\title{
Effect of 3-month Tai Chi Exercise on Heart and Lung Capability of Elder People
}

\author{
Gao Dongqing \\ Department of physical education, Shanghai University of Engineering Science, Shanghai 201620, China \\ qtryxk@126.com
}

\begin{abstract}
To explore the effect of Tai Chi exercise on cardiopulmonary function and physical quality of the elder female individuals. METHODS: The subjects of this experiment are 50 elder female individuals who were divided into 2 groups randomly: the exercise group(25 cases) and the control group(25 cases). The exercise group had learned Tai Chi for one week before the experiment began. After mastering the Tai Chi, the exercise group practiced 50-60min pre time, five times pre week for three months, and the control group had no exercise with collective or individual scheme. The indexes of cardio-pulmonary function and physical quality of the elder female individuals were measured before and after 3-month exercise. RESULTS: After three months Tai Chi exercise, the exercise group showed decrease in $\operatorname{DBP}(\mathrm{P}<0.05)$, increase in FEV1 $(\mathrm{P}<0.05)$, significant increase in FEV1\% $(\mathrm{P}<0.01)$, increase in $\mathrm{FC}, \mathrm{VC}, \mathrm{FVC}$ and $\mathrm{MVV}(\mathrm{P}>0.05)$, significant increase in back strength $(\mathrm{P}<0.01)$, increase in sit-and-reach and eye-closed and single-legged $(\mathrm{P}<0.05)$, significant decrease in reaction time $(\mathrm{P}<0.01)$. The control group showed no significant change in these variables including DBP, FEV1, FEV1\%, back strength, sit-and-reach, eyeclosed and single-legged $(\mathrm{P}>0.05)$. CONCLUSION: Tai Chi exercise has the positive effects on cardio-pulmonary function, and has improved the strength, flexibility, balance and sensitivity and physical qualities of elder female individuals.
\end{abstract}

Index Terms - Tai Chi, Elder people, Heart and lung capability, Physical quality

\section{Introduction}

Tai Chi is one of the Chinese traditional martial arts. Initially, it functioned as a unique and profound self-defense technique. As an ancient Kongfu, Tai Chi includes quan, sword, broadsword, spear, push-hand, etc. Especially the pushhand is well known for its tactic and technique, the powerful and aggressive opponent being defeated by a small clever attack or an abrupt explosive power. As an typical artful Chinese Kongfu, hand push has been highly popular with the small and weak people.

\section{A. Subjects}

The subjects of this experiment are 50 elder female individuals from Donghu Lake District of Shanghai, who are of 61 to 71 years old with an average of $63.4( \pm 2.4)$. The standards for selecting subjects: without recent regular exercise, healthy without disability, with no pills taken which can influence the testing results before or during experiments, knowing and agreeing to the interfering plan and testing item. The subjects of this experiment were divided into 2 groups randomly : the exercise group(25 cases) and the control group( 25 cases $)$. The two groups are not evidently different in age.

\section{B. Methods}

The exercise group had learned Tai Chi for one week before the experiment. After mastering the Tai Chi, the exercise group practiced 50-60min per time, five times per week for three months, and the control group had no exercise with collective or individual scheme. The indexes of cardiopulmonary function and physical quality were measured before and after 3-month exercise.

1) Intervention group: The exercise group had learned Tai Chi for one week before the experiment. After mastering the Tai Chi, the exercise group practiced 50-60min per time, five times per week for three month. The exercise is controlled at intermediate intensity ( MHR 50\% 70\%) .Polar table is applied for supervision. The Tai Chi exercise was organized and executed collectively and instructed by specialists. The control group should not take any planned exercise during the three months.

2) Cardio-pulmonary function and physical quality testing: Cardio-pulmonary function and physical quality were tested before and after 3-month exercise respectively. The indexes of cardio-pulmonary function include faculty of cardio-function(FC), systolic blood pressure ( SBP), diastolic blood pressure (DBP), mean arterial pressure (MAP) ; vital capacity ( VC) , forced vital capacity ( FVC ) ,firstsecond forced exhale volume(FEV1) - forced exhale volume per second (FEV1\%), maximal voluntary ventilation (MVV). The indexes of physical quality: grip power, back strength, sitand-reach, eye-closed and single-legged and reaction time.

3) Statistics methods: SPSS15.0 was employed for data analysis and processing. All data were indicated with the mean \pm standard deviation. Data within groups before and after the experiment were compared with Paired-Samples T Test. Data between groups before and after the experiment were compared with Independent-Samples $\mathrm{T}$ Test. $\mathrm{P}<0.05$ indicated significant differences, and $\mathrm{P}<0.01$ indicated very significant differences.

\section{Results}

A. Changes in cardio-pulmonary function and physical quality of elder female people before and after Tai Chi exercise

From table I, the members of both groups has no obvious 
differences in the indexes of cardio-pulmonary function before the experiment ( $>00.05)$. After three months Tai Chi exercise, the exercise group showed decrease in DBP $(\mathrm{P}<0.05)$, increase in FEV1 $(\mathrm{P}<0.05)$, significant increase in FEV1 \%( $\mathrm{P}<0.01)$, increase in $\mathrm{FC}, \mathrm{VC}, \mathrm{FVC}$ and $\mathrm{MVV}(\mathrm{P}>0.05)$, significant increase in back strength $(\mathrm{P}<0.01)$, increase in sit-and-reach and eye-closed and single-legged $(\mathrm{P}<0.05)$, significant decrease in reaction time $(\mathrm{P}<0.01)$. The control group showed no significant change in these variables including DBP, FEV1, FEV1\%, back strength, sit-and-reach, eye-closed and singlelegged $(\mathrm{P}>0.05)$.

TABLE I Variables changes in cardio-pulmonary function before and after Tai Chi exercise

\begin{tabular}{|l|l|l|l|l|}
\hline \multirow{2}{*}{$\begin{array}{l}\text { Measuring } \\
\text { variables }\end{array}$} & \multicolumn{2}{l|}{ Control Group $(\mathrm{n}=25)$} & \multicolumn{2}{l|}{ Exercise Group $(\mathrm{n}=25)$} \\
\cline { 2 - 5 } & Pre-exercise & Post-exercise & Pre-exercise & Post-exercise \\
\hline FC $($ MET $)$ & $7.47 \pm 1.39$ & $7.53 \pm 1.43$ & $7.28 \pm 1.12$ & $7.83 \pm 1.57$ \\
\hline SBP(mmHg) & $129.47 \pm 9.61$ & $130.21 \pm 8.56$ & $134.34 \pm 7.35$ & $123.32 \pm 8.41$ \\
\hline DBP(mmHg) & $82.88 \pm 4.36$ & $82.05 \pm 5.11$ & $83.71 \pm 5.26$ & $78.09 \pm 4.62 *$ \\
\hline MAP(mmHg) & $97.95 \pm 9.64$ & $98.14 \pm 7.05$ & $98.37 \pm 7.71$ & $92.92 \pm 8.31$ \\
\hline VC(L) & $2.30 \pm 0.63$ & $2.27 \pm 0.23$ & $2.23 \pm 0.42$ & $2.46 \pm 0.55$ \\
\hline FVC(L) & $2.13 \pm 0.19$ & $2.17 \pm 0.29$ & $2.13 \pm 0.21$ & $2.37 \pm 0.23$ \\
\hline FEV1(L) & $1.71 \pm 0.46$ & $1.72 \pm 0.23$ & $1.73 \pm 0.57$ & $2.09 \pm 0.41 *$ \\
\hline FEV1\% & $80.24 \pm 8.77$ & $79.35 \pm 7.91$ & $81.19 \pm 9.16$ & $88.30 \pm 11.34 * *$ \\
\hline MVV(L/min) & $75.26 \pm 9.91$ & $75.53 \pm 9.32$ & $77.17 \pm 8.05$ & $82.03 \pm 9.29$ \\
\hline
\end{tabular}

Compared with Pre-exercise, ${ }^{*} \mathrm{P}<0.05, * * \mathrm{P}<0.01$; Compared with Postexercise \# $\mathrm{P}<0.05$, \# \# $\mathrm{P}<0.01$.

\section{B. Changes in physical quality of elder female people before and after Tai Chi exercise}

From table II, the members of both groups has no obvious differences in the indexes of physical quality before the experiment ( $p>0.05)$. After three months Tai Chi exercise, the exercise group showed significant increase in back strength $(\mathrm{P}<0.01)$, increase in sit-and-reach and eye-closed and singlelegged $(\mathrm{P}<0.05)$, significant decrease in reaction time $(\mathrm{P}<0.01)$, significant increase in grip power $(\mathrm{P}>0.05)$. The control group showed no significant change in these variables of physical quality.

TABLE II Variables changes in physical quality before and after Tai Chi exercise

\begin{tabular}{|c|l|l|l|l|}
\hline \multirow{2}{*}{$\begin{array}{c}\text { Measuring } \\
\text { variables }\end{array}$} & \multicolumn{2}{|l|}{ Control Group $(\mathrm{n}=25)$} & \multicolumn{2}{l|}{ Exercise Group $(\mathrm{n}=25)$} \\
\cline { 2 - 5 } & Pre-exercise & Post-exercise & Pre-exercise & Post-exercise \\
\hline $\begin{array}{c}\text { Grip power } \\
(\mathrm{kg})\end{array}$ & $21.15 \pm 3.78$ & $21.39 \pm 3.57$ & $20.63 \pm 4.21$ & $21.69 \pm 4.73$ \\
\hline $\begin{array}{c}\text { Back strength } \\
(\mathrm{kg})\end{array}$ & $317.5 \pm 111.7$ & $315.9 \pm 118.3$ & $329.9 \pm 108.5$ & $449.5 \pm 112.4^{* *}$ \\
\hline $\begin{array}{c}\text { Sit-and-reach } \\
(\mathrm{cm})\end{array}$ & $5.53 \pm 9.11$ & $5.55 \pm 8.19$ & $5.87 \pm 8.42$ & $7.87 \pm 9.34^{*}$ \\
\hline $\begin{array}{c}\text { Eye-closed ands } \\
\text { ingle-legged } \\
(\mathrm{s})\end{array}$ & $7.71 \pm 8.67$ & $7.99 \pm 7.93$ & $7.26 \pm 8.17$ & $9.92 \pm 10.13^{*}$ \\
\hline $\begin{array}{c}\text { reaction time } \\
(\mathrm{s})\end{array}$ & $0.21 \pm 0.02$ & $0.21 \pm 0.03$ & $0.22 \pm 0.02$ & $0.18 \pm 0.04^{* *}$ \\
\hline
\end{tabular}

Compared with Pre-exercise, ${ }^{*} P<0.05,{ }^{*}{ }^{*} P<0.01$; Compared with Postexercise $^{\#} P<0.05,{ }^{\#} P<0.01$.

\section{Discussions}

A. Effect of Tai Chi on cardio-pulmonary function and physical quality of elder female people

With age added, Cardio-pulmonary systems become old and feeble, featuring some changes such as hardened arteries vessels, deceasing elastic obedience of blood vessel, increasing resistance outside blood vessels, higher blood pressure, blocked function of respiratory muscle, lower respiratory work ${ }^{[1,2]}$. Researches and relative studies has shown that exercises such as walking, jogging, Ba Duan Jin and Taiji can effectively improve the cardio-pulmonary function of elder people. Du Shaowu has found that after 6-month exercise of Tai Chi, the subjects have significantly larger SV and higher early peak value in flowing speed of mitral diastolic than those of pre-exercise, suggesting an improvement in ventricular systolic and diastolic function ${ }^{[3]}$. This study uses Tai Chi as a main exercise method, and gets results as below: after 3month exercise, elder female people have a significant increase in BDP and FC and SBP, MAP and PP decrease accordingly. Such results are generally complied by the researches by scholars listed above. What's more, This study has found that after 3-month exercise, elder female people have a significant increase in FEV1 and FEV1\%, and VC and FVC has improved accordingly. Foreign scholars Albouaini ${ }^{[4]}$ and Guazzi ${ }^{[5]}$ have also made researches and held that reasonable exercise can higher FEV1 and FEV1 of elder people and lower their blood pressure.

By the results of this experiments, a conclusion is that elder female people can obtain effective improvement in cardio-pulmonary function by 3-month exercise of Tai Chi. The possible causes can be various. From view of traditional Chinese Medicine, Tai Chi mediates the blood vessel nets and air-breath more fluent and enhances the blood recycle by body instruction; mediates the functions of internal organs of body by the drawing exercise led by blood vessel nets; improve the cardio-functions by stimulating the inner air and adding force the spiritually and physically. From view of the features of natural respiratory of Tai Chi, during various actions of the exercise, many parts of muscles have take part in the, making respiratory an initiate one and that is not only conducive to the incensement of shrinkage force, endurance and flexibility of respiratory muscles but also imposes good effects on the more openness of Alveolar and elasticity of lungs. From perspective of improving nerve system, 3-month exercise of Tai Chi has improved functions of automatic nerve and makes cardiovascular mechanism have changes for better adjustment.

\section{B. Effect of Tai Chi on Physical Quality of Elder Female People}

With the age growing, the elder people tend to have a fall in their physical qualities including strength, flexibility, balance and sensibility. Proper exercise, as a relevant research report believes, can help delay the drop of the elder people's physical qualities. As Zeng Yungui has discovered, after 75 days' Ba Duan Jin exercise, gain a pronounced climb in grip power, lower limbs strength, sit-and-reach, and eye-closed 
and single-legged as well as a remarkably shortened reaction time $^{[6]}$. According to the research by Cheng Qilian, the elder and mid-age people after a year's Tai Chi exercise achieve a notable promotion in grip power, lower limbs strength, sit-andreach, and eye-closed and single-legged, and acquire an shorter reaction time ${ }^{[7]}$. As Zhu Hanxiao observes, 30 elder female people after 16-weeks exercise of 5 Bird Game exercise has a greatly shortened reaction time and a notable improvement in eye-closed and single-legged ${ }^{[8]}$. The results by the scholars mentioned above has been further proved in this research which finds that the elder female people get a better back strength, perform better in sit-and-reach and eye-closed and single-legged and shorter reaction time after 3 months' Tai Chi exercise.

From this research, the reason why doing Tai Chi exercise could obviously improve their physical qualities such as grip power, back strength, sit-and-reach, eye-closed and single-legged, and reaction time may closely relates to the property of Tai Chi to change in bone and muscle. In each action of Tai Chi, forearms, lower limbs and the body fully shrink, stretch and spin, by which all joints of the body get multidirectional movements to Stretch the body's big and small muscle groups and fascias and connective tissues around joints like muscle tendons, ligamenta and joint capsules and by which the improvement is achieved in blood circulation of soft tissues in the movable parts, the nutrition metabolizing of the soft tissues, raising capacity of nerve to masclesand proprioceptive ability, inflexibility and sensibility of the soft tissues like muscles, muscle tendons and ligamenta, the moving ability of tissues like bones, joints and muscles so as to reach the goal of building better physical qualities.

\section{Conclusion}

After 3 months' Tai Chi exercise the elder female individuals have an obvious drop in DBP, and rise in both FEV1 and FEV1\%. They also achieve marked promotion in back strength, sit-and-reach and eye-closed and single-legged with shorter reaction time. It indicates that Tai Chi could improve cardiovascular elasticity of the elder, lowering blood pressure, promoting pulmonic recoil force, respiratory muscle strength and respiratory reserve capacity and enhancing the physical qualities including strength, flexibility, balance and sensibility. 3-month Tai Chi exercise has produced positive effects on the physical mechanism of cardiovascular and respiratory systems and physical quality.

\section{References}

[1] Lang CC, Agostoni P, Mancini DM. Prognostic significance and measurement of exercise-derived hemodynamic variables in patients with heart failure. J Card Fail. 2007 Oct; 13(8):672-679.

[2] Lin Wei, Jiang Xiao-Mao, Bo Yao, et al. Different intensity exercise on the cardiopulmonary function of the elderly. Chinese Journal of Physical Medicine and Rehabilitation, 2006,28 (7) :440-441.

[3] Du Shao-Wu. Yijin Jing Qigong exercise on cardiac function in the elderly. Chinese Journal of Sports Medicine, 2006, 25 (6):721-722.

[4] Albouaini K, Egred M, Alahmar A, Wright DJ. Cardiopulmonary exercise testing and its application. Heart. 2007 Oct; 93(10):1285-1292.

[5] Guazzi M, Myers J, Arena R. Cardiopulmonary exercise testing in the clinical and prognostic assessment of diastolic heart failure. J Am Coll Cardiol. 2005 Nov 15; 46(10):1883-1890.

[6] has the Yunnan-Guizhou, ZHOU Xiao-qing, Wang Amway, et al. • Ba Duan Jin Qigong exercise on the elderly health effects of morphology and physiological studies. Journal of Beijing University of Physical Education, 2005, 28 (9): 1207 -1209.

[7] Cheng, training, Du Shao-wu, Zhang Wen-chun, et al. Health Qigong • Yi Jinjing physical exercise on the impact of the elderly. Journal of Beijing Sport University, 2006, 29 (11) :1516-1517.

[8] Zhu Han Xiao, Jia Yong Sun, Xue-Lian Chen. A New 16-week exercise on five birds show older women related indicators of bodily functions. Chinese Journal of Sports Medicine, 2008, 27 (4):499-500. 